\title{
TUMORES DO PLEXO CORÓIDEO
}

\author{
GERALDO PIANETTI*, LUIZ FERNANDO FONSECA **
}

\begin{abstract}
RESUMO - É feita análise estatística, semiológica, terapêutica e da evolução de 15 crianças portadoras de tumor do plexo coróideo, tratadas cirurgicamente no Serviço de Neuropediatria dos Hospitais das Clínicas (UFMG) e São Francisco de Assis, num período de 15 anos (1981 a 1996). A idade variou de quatro meses a 16 anos, com média de três anos e seis meses, dez tinham menos de dois anos; nove eram do sexo feminino; 14 tinham sinais de hipertensão intracraniana; em todas elas a presença do tumor foi confirmada pelo estudo com tomografia computadorizada; em oito crianças o tumor estava localizado em um ventrículo lateral. Das 15 crianças, 11 necessitaram de derivação ventrículo-peritoneal; a exerese radical do tumor foi possível em 13 das 14 crianças operadas. O diagnóstico anátomo-patológico foi papiloma em 12 crianças e carcinoma nas outras duas. Duas crianças faleceram, uma no sétimo dia de pós-operatório e a outra 12 meses após, com metástases disseminadas pelo sistema nervoso central. É feita revisão da literatura pertinente e dados são comparados.
\end{abstract}

PALAVRAS-CHAVE: papiloma do plexo coróideo, carcinoma do plexo coróideo.

\section{Choroid plexus tumors}

ABSTRACT - This analysis comprises 15 children under 16 years of age, with choroid plexus tumors, seen in the Service of Paediatric Neurosurgery, Hospital das Clínicas and Hospital São Francisco de Assis in Belo Horizonte, Brazil, between 1981 and 1996. The patients were aged between 4 months and 16 years (average of 3 years and a half); 10 were less than 2 years, 9 were female; 14 children had clinical evidence of intracranial hypertension. All the children underwent CT scan and the choroid plexus tumors were clearly demonstrated in 14 of then. In 8 children the tumors were located in one lateral ventricle, 5 in the fourth ventricle and 2 had the tumors in more than one ventricle, 11 children required ventriculo-peritoneal shunt; 14 cases were operated on, 13 with total excision; 2 children died, respectively 7 days and one year after the surgery. Pathological examination revealed papillomas in 12 cases and carcinoma in two cases.

KEY WORDS: choroid plexus papilloma, choroid plexus carcinoma.

As lesões tumorais do plexo coróideo são raras, representando, em todas as idades, de $0,4 \%$ a $0,6 \%$ dos tumores intracranianos e de $1,0 \%$ a 4,0\% dos tumores intracranianos até os 15 anos de idade $^{1-6}$. Esta incidência observada nas crianças aumenta quando se analisam menores de dois anos de idade, faixa etária em que aparece a maioria dos tumores desta região $0^{3,4,7-9}$. Dentre os tumores, o mais comum é o papiloma, seguido pelo carcinoma do plexo coróideo que ocorre entre 20,0 e 40,0\% dos $\operatorname{casos}^{3,7,10}$; raramente é encontrada hipertrofia do plexo coróideo com sintomatologia tumoral. Segundo Raimondi e Gutierrez ${ }^{6}$, a primeira lesão tumoral do plexo coróideo foi descrita por Guerard em 1833; VonPlath em 1884, Vigoureaux em 1908 e DeMange em 1924 foram os primeiros a relacionar o tumor do plexo coróideo com hidrocefalia; a primeira ressecção cirúrgica foi feita em 1906, por Bielschowsky e Unger, o paciente evoluindo para o óbito no pós-operatório imediato; Pether, em 1919, relata a primeira ressecção cirúrgica de tumor do plexo coróideo de ventrículo

Serviço de Neurocirurgia Pediátrica dos Hospitais das Clínicas (UFMG) e São Francisco de Assis, Belo Horizonte, Brasil: *Neurocirurgião, Professor Adjunto da Faculdade de Medicina da Universidade Federal de Minas Gerais (UFMG); **Neuropediatra. Aceite: 7-fevereiro-1998.

Geraldo Pianetti - Rua dos Aimorés 2480/902 - 30140-072 Belo Horizonte MG - Brasil. 
lateral, com sucesso; em 1922, Sachs retira, com sucesso, um papiloma do plexo coróideo do quarto ventrículo; o mesmo ocorreria com Cushing em 1923. A primeira grande série de tumores do plexo coróideo em crianças foi apresentada por Matson e Crofton 4, em 1960.

A patogênese da hidrocefalia que geralmente acompanha estas lesões, o momento e a necessidade de derivação ventricular, a técnica e tática cirurgicas e o tratamento complementar são questões que merecem ser analisadas à luz de experiências contemporrâneas. É feita análise da clínica, do tratamento e da evolução de quinze crianças portadoras de tumores do plexo coróideo. A análise é homogênea, pois todas as crianças foram operadas pelo mesmo cirurgião e acompanhadas pelo mesmo neuropediatra.

\section{CASUÍSTICA}

A experiência pessoal com os tumores do plexo coróideo em crianças, consta de 15 pacientes tratados entre 1981 e 1996, no Serviço de Neurocirurgia Pediátrica dos Hospitais das Clínicas (UFMG) e São Francisco de Assis, em Belo Horizonte (Tabela 1). Neste mesmo período, foram tratadas, pelos autores, 157 crianças com tumores intracranianos. A incidência de crianças com tumores do plexo coróideo foi $9,5 \%$. Nove crianças eram do sexo feminino $(60,0 \%)$ e seis do sexo masculino $(40,0 \%)$. A idade variou de quatro meses a 16 anos, com média de três anos e seis meses; dez crianças $(66,6 \%)$ tinham menos de dois anos, das quais seis $(40,0 \%)$ tinham menos de um ano; duas $(13,3 \%)$ estavam entre dois e cinco anos e três $(20,0 \%)$ com mais de cinco anos.

Tabela 1. Relação dos pacientes com tumores do plexo coróideo.

\begin{tabular}{|c|c|c|c|c|c|c|c|c|c|c|}
\hline Caso & Nome & Idade/Sexo & Data & Clínica & Localização & Cirurgia & $\mathrm{AP}$ & DVP & Rxt/Qxt & $\begin{array}{c}\text { Evolução/ } \\
\text { Tempo }\end{array}$ \\
\hline 1 & SGJ & 2 a $2 \mathrm{~m} / \mathrm{M}$ & 1981 & HIC/As & VLD & Radical & $\mathrm{Pp}$ & Pré & & $\mathrm{OK} / 180 \mathrm{~m}$ \\
\hline 2 & GNB & $4 \mathrm{~m} / \mathrm{M}$ & 1981 & HIC & VLD & Radical & $\mathrm{Pp}$ & Pré & & $\mathrm{OK} / 180 \mathrm{~m}$ \\
\hline 3 & EM & $15 \mathrm{a} / \mathrm{F}$ & 1982 & HIC/At & IV & Radical & $\mathrm{Pp}$ & & & $\mathrm{OK} / 168 \mathrm{~m}$ \\
\hline 4 & JFVA & $2 \mathrm{a} / \mathrm{M}$ & 1984 & HIC/At & IV & Radical & $\mathrm{Pp}$ & Pré & & $\mathrm{OK} / 144 \mathrm{~m}$ \\
\hline 5 & RAT & $2 \mathrm{a} / \mathrm{M}$ & 1984 & HIC/Ds & IV & Radical & $\mathrm{Pp}$ & Pré & & $\mathrm{OK} / 159 \mathrm{~m}$ \\
\hline 6 & LMM & $6 \mathrm{~m} / \mathrm{M}$ & 1984 & HIC & VLE & Radical & $\mathrm{Pp}$ & Pré & & $\mathrm{OK} / 159 \mathrm{~m}$ \\
\hline 7 & KVB & $9 \mathrm{~m} / \mathrm{M}$ & 1989 & $\mathrm{HIC}$ & VLE+III & Recusa & $?$ & Pré & & S/A \\
\hline 8 & RRM & $3 \mathrm{a} / \mathrm{M}$ & 1991 & Ataxia & VLE & Radical & $\mathrm{Pp}$ & & & $\mathrm{OK} / 74 \mathrm{~m}$ \\
\hline 9 & LAF & $6 \mathrm{~m} / \mathrm{F}$ & 1994 & $\mathrm{HIC}$ & VLE & Radical & $\mathrm{Ca}$ & & & $\begin{array}{c}\text { Hemiparesia } \\
35 \mathrm{~m}\end{array}$ \\
\hline 10 & IPL & $9 \mathrm{~m} / \mathrm{F}$ & 1994 & $\mathrm{HIC} / \mathrm{Cv}$ & VLE & Radical & $\mathrm{Ca}$ & Pré & Rxt/Qxt & Óbito/12m \\
\hline 11 & ILMC & 1 a $9 \mathrm{~m} / \mathrm{F}$ & 1995 & $\mathrm{HIC}$ & IV & Radical & $\mathrm{Pp}$ & & & $\mathrm{OK} / 28$ \\
\hline 12 & LVD & 1 a $6 \mathrm{~m} / \mathrm{F}$ & 1995 & $\begin{array}{l}\mathrm{HIC} / \mathrm{Cv}+ \\
\text { atraso }\end{array}$ & $\begin{array}{c}\text { VLE+VLD } \\
+ \text { III }\end{array}$ & Radical & $\mathrm{Pp}$ & Pós & & $\begin{array}{l}\text { Atraso+ } \\
\mathrm{Cv} / 24 \mathrm{~m}\end{array}$ \\
\hline 13 & VVO & $9 \mathrm{a} / \mathrm{F}$ & 1995 & HIC/At & IV & Parcial & $\mathrm{Pp}$ & Pré & & Obito/7dias \\
\hline 14 & PCB & $7 \mathrm{~m} / \mathrm{F}$ & 1996 & HIC/As & VLD & Radical & $\mathrm{Pp}$ & Pré & & $\mathrm{OK} / 16 \mathrm{~m}$ \\
\hline 15 & $\mathrm{AAC}$ & $16 \mathrm{a} / \mathrm{F}$ & 1996 & $\mathrm{HIC}$ & VLE & Radical & $\mathrm{Pp}$ & Pré & & $\mathrm{OK} / 9 \mathrm{~m}$ \\
\hline
\end{tabular}

Legenda: M, masculino; F, feminino; HIC. hipertensão intracraniana; As, ascite; At. ataxia de marcha; Ds, dismetria; Cv, convulsão; VDL, ventrículo lateral direito; VLE, ventrículo lateral esquerdo; III, terceiro ventrículo; IV, quarto ventrículo; AP, anatomia patológica; Pp, papiloma; Ca, carcinoma; DVP, derivação ventrículo-peritoneal; Rxt, radioterapia; Qxt, quimioterapia; OK, paciente com exame neurológico e tomografia normais; S/A, sem acompanhamento. 
Das 15 crianças, $14(96,0 \%)$ se apresentaram com evidencias clínicas de hipertensão intracraniana, sendo oito com aumento do perímetro craniano; três (20,0\%) tiveram convulsões associadas; quatro (26,6\%) apresentaram ataxia de marcha, sendo que em uma delas este era o único sintoma e em duas crianças, nas quais o diagnóstico inicial era só de hidrocefalia, as manifestações de hipertensão intracraniana se associaram a presença de ascite. Somente uma criança apresentava malformação associada, caracterizada pela síndrome de Aicardi (criança de sexo feminino, atraso no desenvolvimento neuropsicomotor, convulsões do tipo espasmo em flexão, coriorretinite lacunar e agenesia do corpo caloso). Todas as crianças se submeteram a estudo tomográfico (TC) do encéfalo, (Fig 1) com contraste, sendo a lesão bastante evidente em 14 delas, se caracterizando por tumoração que capta contraste, localizada no interior de um dos ventrículos; duas crianças tiveram o estudo semiológico completado com a ressonância nuclear magnética (RNM). Treze crianças $(86,6 \%)$ tinham evidencias radiológicas de hidrocefalia associada à lesão do plexo coróideo. Em 13 crianças $(86,6 \%)$ a lesão estava confinada a uma cavidade ventricular, sendo cinco no ventrículo lateral esquerdo (33,3\%), três no ventrículo lateral direito $(20,0 \%)$ e cinco no quarto ventrículo $(33,3 \%)$; nos outros casos $(13,4 \%)$ havia acometimento de mais de uma cavidade ventricular, sendo uma criança com tumor no ventrículo lateral esquerdo e terceiro ventrículo e outra com a lesão ocupando ambos os ventrículos laterais e o terceiro ventrículo.

Das 13 crianças que tinham evidencias radiológicas e clínicas de hidrocefalia, dez receberam derivação ventrículo-peritoneal no pré-operatório e uma no pós-operatório. O tratamento foi cirúrgico em 14 crianças $(93,3 \%)$, com ressecção radical da massa tumoral em 13 delas; uma criança não teve o tumor ressecado por recusa de familiares, só recebeu a derivação ventricular.

O exame anátomo-patológico mostrou papiloma benigno em 12 crianças $(80,0 \%)$ e carcinoma em duas $(13,3 \%)$; houve forte suspeita radiológica de papiloma na criança que não foi operada devido a recusa de familiares. Uma criança, portadora de carcinoma do plexo coróideo já disseminado, recebeu tratamento complementar com radioterapia e quimioterapia.

Das 12 crianças portadoras de papiloma benigno do plexo coróideo e tratadas cirurgicamente, 11 estão vivas (evolução de nove a 180 meses, com média de 89,08 meses), das quais dez com exame neurológico normal e uma com atraso no desenvolvimento neuropsicomotor e convulsões; uma faleceu no pós-operatório imediato. Das duas crianças portadoras de carcinoma do plexo coróideo, uma apresenta discreta hemiparesia e tomografia normal após três anos da cirurgia e a outra faleceu com metátases disseminadas no sistema nervoso central (SNC), um ano após a cirurgia, apesar do tratamento complementar agressivo que foi instituído, com radioterapia e quimioterapia. $\mathrm{O}$ tempo de acompanhamento de todos os pacientes variou de nove meses a 15 anos com média de 79,7 meses.

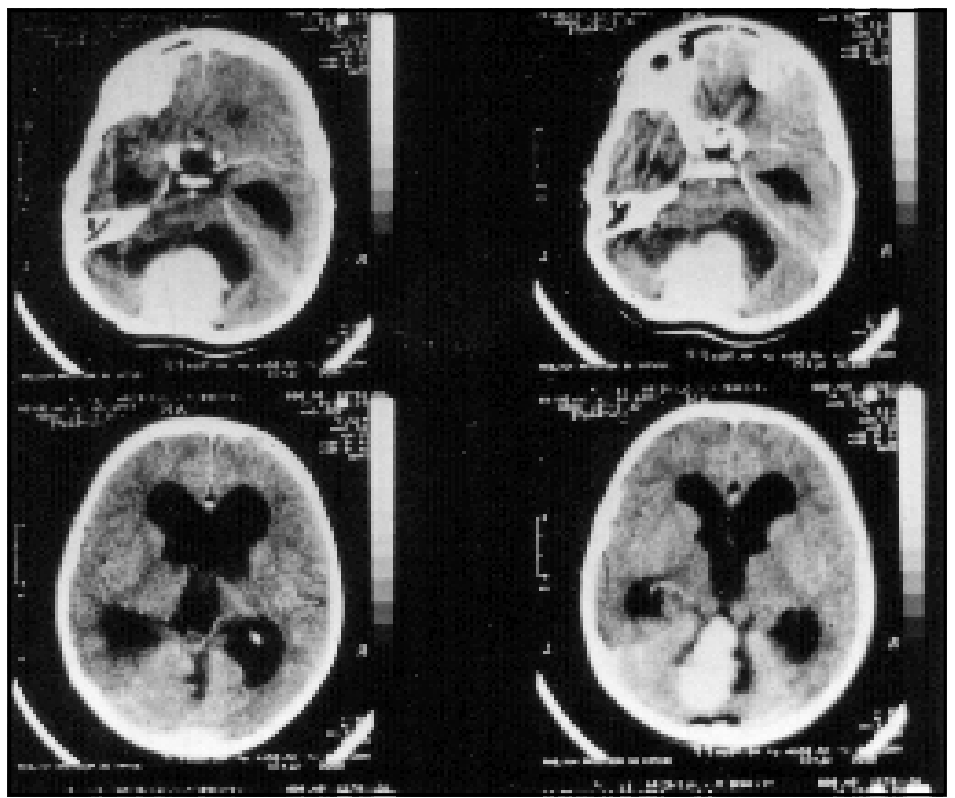

Fig 1. TC mostrando papiloma do plexo coróideo localizado no quarto ventrículo. 


\section{DISCUSSÃO}

Não existe, na literatura consultada, uma larga experiência com o tratamento de tumores do plexo coróideo em crianças até 16 anos; vários serviços têm publicado estudos com um número reduzido de casos; Matson e Crofton ${ }^{4}$, em 1960, publicaram a primeira série com 16 casos, Wilkins e Rutledge ${ }^{11}$, em 1961, apresentaram 12 casos, Raimondi e Gutierrez ${ }^{6}$, em 1975, analisaram sua experiência com 22 crianças operadas, Tomita e McLone ${ }^{9}$, em 1985, apresentaram 17 casos; Lena e col. $^{3}$ mostraram a experiência européia em 1990, com 24 crianças operadas; e, finalmente, em 1991, StClair e col. ${ }^{10}$ apresentaram a experiência do Serviço de Chicago com 28 casos coletados em 33 anos. Muitos dos pacientes citados nesses estudos foram operados antes de 1975 e observam-se divergências importantes na análise das conclusões, uma vez que o tratamento e resultado modificaram muito após o advento de métodos diagnósticos mais precisos como a TC e a RNM, além da modernização de técnicas anestésicas e a microcirurgia. Isto faz com que dúvidas permaneçam na conduta destes tumores.

As lesões tumorais do plexo coróideo se manifestam, de preferência, nas crianças até os 16 $\operatorname{anos}^{11}$ e, entre crianças, varia de 3,0 a 7,5\% $\%^{3,4,6,12,13}$. São bastante mais frequentes antes dos dois anos de idade, atingindo de 60 a $80 \%{ }^{3,4,8}$. Na experiência pessoal, os tumores do plexo coróideo atingiram 9,5\% (15/157) de todos os tumores intracranianos em crianças, acima das estatisticas mundiais, sendo que dez $(66,6 \%)$ tinham menos de 24 meses.

Não existe diferença, estatisticamente significante, em relação à incidência entre os sexos masculino e feminino ${ }^{3,4,11}$. No presente estudo foi observada ligeira predominância no sexo feminino com nove crianças $(60,0 \%)$ entre as 15 estudadas.

A localização preferencial dos tumores do plexo coróideo em crianças é no ventrículo lateral, o que ocorre entre 45 a $85 \%$ dos $\operatorname{casos}^{3,4,6,8}$ e difere do adulto, que tem a localização preferencial no quarto ventrículo ${ }^{14,15}$. Existe ligeira preferencia pelo ventrículo lateral esquerdo sobre o direito. No terceiro ventrículo são encontrados entre 7 a $29 \%$ dos $\operatorname{casos}^{3,4,8}$ e no quarto ventrículo entre 10 a $20 \%{ }^{3}$. Nas crianças, a localização primária no ângulo ponto cerebelar é rara, o mesmo acontecendo com a localização em mais de um ventrículo. Dos casos de Lena e col. ${ }^{3}, 11$ dos 24 tumores estavam nos ventrículos laterais (seis no esquerdo e cinco no direito), sete estavam no terceiro ventrículo e seis no quarto ventrículo; na experiência da Clínica $\mathrm{Mayo}^{14}$, todas as sete crianças tinham o tumor localizado no ventrículo lateral e Raimondi e Gutierrez ${ }^{6}$ apresentaram estatística com 86,9\% dos casos localizados em um ventrículo lateral. Os relatos de tumores do plexo coróideo acometendo os dois ventrículos laterais são eventuais, aparecem na literatura em 1884 com Demselben, Cushing ${ }^{2}$ em 1925, Ray em 1956, Matson ${ }^{4}$ em 1960 e Knierim ${ }^{8}$ em 1991. Não foi encontrado na literatura consultada um caso de criança operada em que existissem tumores nos ventrículos laterais e no terceiro ventrículo. Na experiência pessoal, dos 15 pacientes analisados, oito $(53,3 \%)$ tinham os tumores isolados nos ventrículos laterais, cinco $(33,3 \%)$ no quarto ventrículo, um $(6,7 \%)$ com tumores no ventrículo lateral e terceiro ventrículo e um caso raro com tumores em ambos os ventrículos laterais e no terceiro ventrículo (Fig 2). Assim como Raimondi e Gutierrez ${ }^{6}$, não tivemos nenhum caso com tumor isolado no terceiro ventrículo, nem no ângulo-ponto-cerebelar.

A sintomatologia dos tumores do plexo coróideo é inespecífica e vai depender da localização. Quando nos ventrículos laterais e terceiro ventrículo, se manifestam com quadro clássico de hipertensão intracraniana com cefaléia, vômitos e papiledema nas crianças acima dos dois anos e macrocrania nas crianças com menos de dois anos. Na literatura ${ }^{3,4}$ encontra-se porcentagem de 70,0\% dos casos com sinais de hipertensão intracraniana. Quando acometem o quarto ventrículo, sinais cerebelares, geralmente ataxia de marcha ou astasia, se associam ao quadro de hipertensão intracraniana. Outros sinais e sintomas podem eventualmente aparecer, tais como sinal de Parinaud, convulsões, déficits motores e atraso no desenvolvimento neuropsicomotor ${ }^{3}$. Dos 24 casos de Lena e col. ${ }^{23} 18(75,0 \%)$ apresentavam sinais de hipertensão intracraniana, sendo dez $(41,6 \%)$ com 


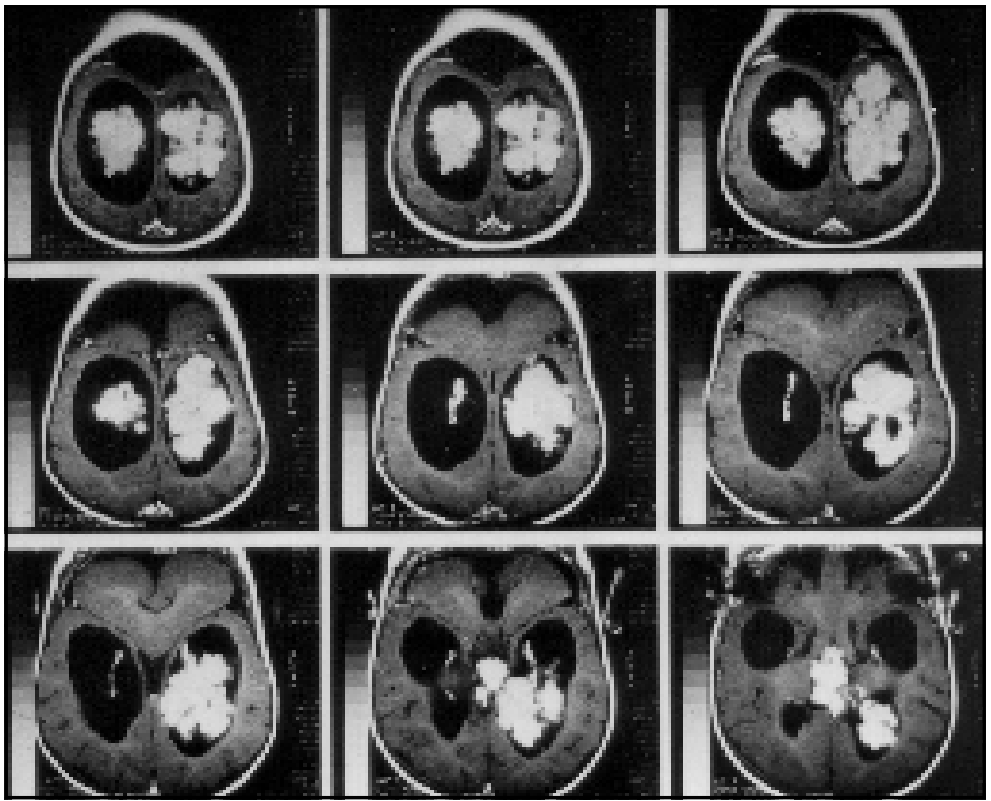

Fig 2. RNM mostrando papiloma do plexo coróideo localizado no terceiro ventrículo e nos ventrículos laterais. Paciente portador da síndrome de Aicardi.

macrocrania; cinco tinham atraso no desenvolvimento neuropsicomotor. Dos oito casos de Kinierim ${ }^{8}$, cinco $(62,5 \%)$ apresentavam macrocrania. Todas as crianças com tumor do plexo coróideo, tratadas na Clínica $\mathrm{Mayo}^{14}$, apresentavam sinais de hipertensão intracraniana. Matson e Crofton ${ }^{4}$ mostram que $16(69,5 \%)$ dos 23 pacientes tinham aumento do perímetro cefálico e $11(47,8 \%)$ tinham papiledema. Na experiência pessoal, $14(96,0 \%)$ apresentavam sinais de hipertensão intracraniana, sendo oito $(53,3 \%)$ com macrocrania; duas crianças apresentavam, além dos sinais de hipertensão intracraniana, quadro de ascite importante; o mesmo ocorreu em casos de Kinierim ${ }^{8}$. Foram crianças encaminhadas ao serviço com o diagnóstico único de hidrocefalia e mal funcionamento valvular. Um de nossos pacientes, do sexo feminino, tinha quadro de atraso no desenvolvimento neuropsicomotor associado a coriorretinite lacunar e convulsões do tipo espasmos em flexão, confirmando o diagnóstico de síndrome de Aicardi ${ }^{16}$; devido a aumento do perímetro cefálico foi submetida a estudo tomográfico que revelou agenesia do corpo caloso e presença de tumores no terceiro ventrículo e nos ventrículos laterais direito e esquerdo; foi operada e o exame revelou ser papiloma do plexo coróideo. Esta associação é rara, com cerca de dez casos descritos na literatura mundial ${ }^{17-19}$ e questionase se o papiloma do plexo coróideo não seria parte integrante da síndrome de Aicardi.

A comprovação diagnóstica se faz por de imagem, como a TC e a RNM. Geralmente, o estudo pela TC é suficiente para confirmar o diagnóstico, mostrando o grau de hidrocefalia, a localização tumoral, se o tumor está confinado à cavidade ventricular ou se invade o parênquima cerebral, além de possíveis disseminações no SNC. A RNM trouxe subsídios importantes, localiza extensões extraventriculares do tumor e, certamente, a angiorressonância traz melhor visibilização do pedículo vascular. A experiência no diagnóstico dos papilomas do plexo coróideo com a RNM ainda é pequena. A ultra-sonografia trans-fontanela ${ }^{3,20}$ em crianças com menos de um ano pode mostrar o grau de hidrocefalia e a presença de massa tumoral no ventrículo lateral, porém não dispensa o estudo tomográfico antes da cirurgia. A angiografia cerebral mostra a vascularização do tumor, foi muito importante no diagnóstico antes da era da tomografia ${ }^{6}$, mas atualmente está com 


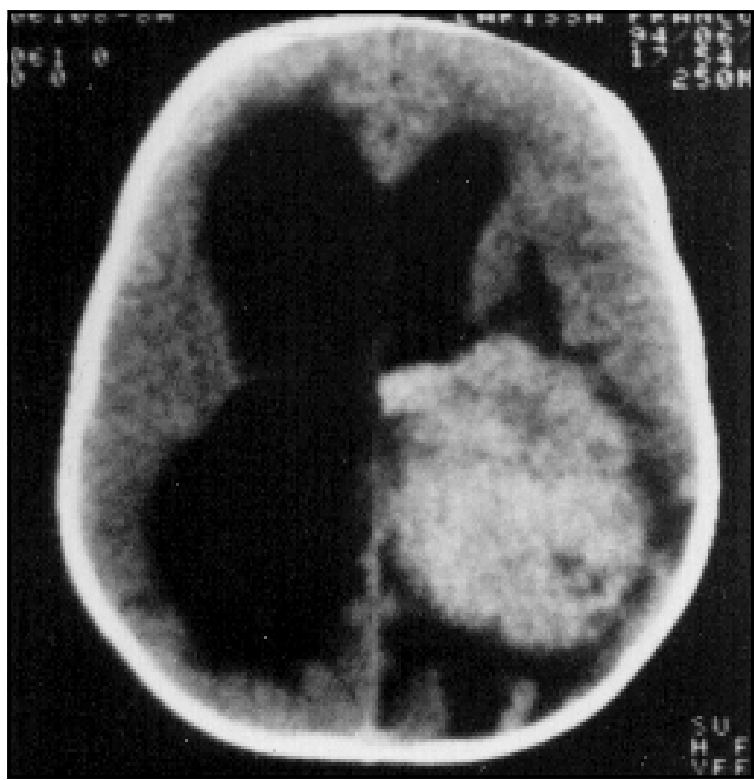

Fig 3. TC pré-operatória de paciente com carcinoma do plexo coróideo no ventrículo lateral, submetida a tratamento cirúrgico e com sobrevida superior a três anos.

seu lugar na história pois não traz qualquer benefício ao paciente ${ }^{3}$, além das dificuldades técnicas na sua realização. Na experiência pessoal, todas as crianças foram submetidas ao estudo tomográfico antes do tratamento cirúrgico e, em somente um caso, o tumor do plexo coróideo não foi bem demonstrado; a presença de hidrocefalia foi observada em 13 das 15 crianças examinadas; duas tiveram a propedêutica complementada com a RNM: em uma delas o tumor foi bem individualizado em três cavidades ventriculares (terceiro ventrículo e ventrículos laterais), com irrigação independente, e no outro caso, em que a TC foi inconclusiva, pode ser bem definida pequena tumoração no plexo coróideo de um dos ventrículos laterais.

A hidrocefalia está usualmente presente nos pacientes com tumores do plexo coróideo ${ }^{3,4,6,10}$. Existem relatos esporádicos de crianças com esta patologia e sem hidrocefalia ${ }^{21,22}$. Desde estudos de Russell, em 1941, a fisiopatologia da hidrocefalia que acompanha estes tumores tem sido discutida e parece que múltiplos fatores podem ser responsabilizados, tais como a hiperprodução do líquor pelo tumor ${ }^{23-25}$, a obstrução do fluxo liquórico pelo próprio tumor (obstrução do forame de Monro, terceiro ou quarto ventrículo) ou a dificuldade na reabsorção devido a fibrose como consequência de repetidas hemorragias ou mesmo descamação tumoral ${ }^{25}$. Vários estudos defendem as duas teorias. A hiperprodução de líquor é constatada através da observação de que, em alguns casos, a hidrocefalia desaparece após a cirurgia com retirada total da lesão, ${ }^{4,14,26-28}$. Outros autores s $^{3,6,25,28,29}$ citam casos em que, apesar da retirada do tumor, persistiu a hidrocefalia. Interessante é que existem divergências quanto ao uso de derivações: Matson e Crofton ${ }^{4}$, em 1960, afirmavam que existiam fortes evidências de que a hidrocefalia era causada unicamente pela hiperprodução de líquor pelo tumor e a afirmativa se baseava no fato de que em todas as crianças em que um papiloma do plexo coróideo do ventrículo lateral foi extirpado totalmente, não se fez necessário o uso de derivações; Raimondi e Gutierrez ${ }^{6}$, em 1976, relatam da necessidade de derivações ventriculares definitivas em 16 (69,5\%) das 23 crianças submetidas a retirada do tumor do plexo coróideo e Lena e col. ${ }^{3}$ em 1990 relatam que 17 $(70,8 \%)$ das 24 crianças submetidas a ressecção de tumor do plexo coróideo necessitaram de derivação 


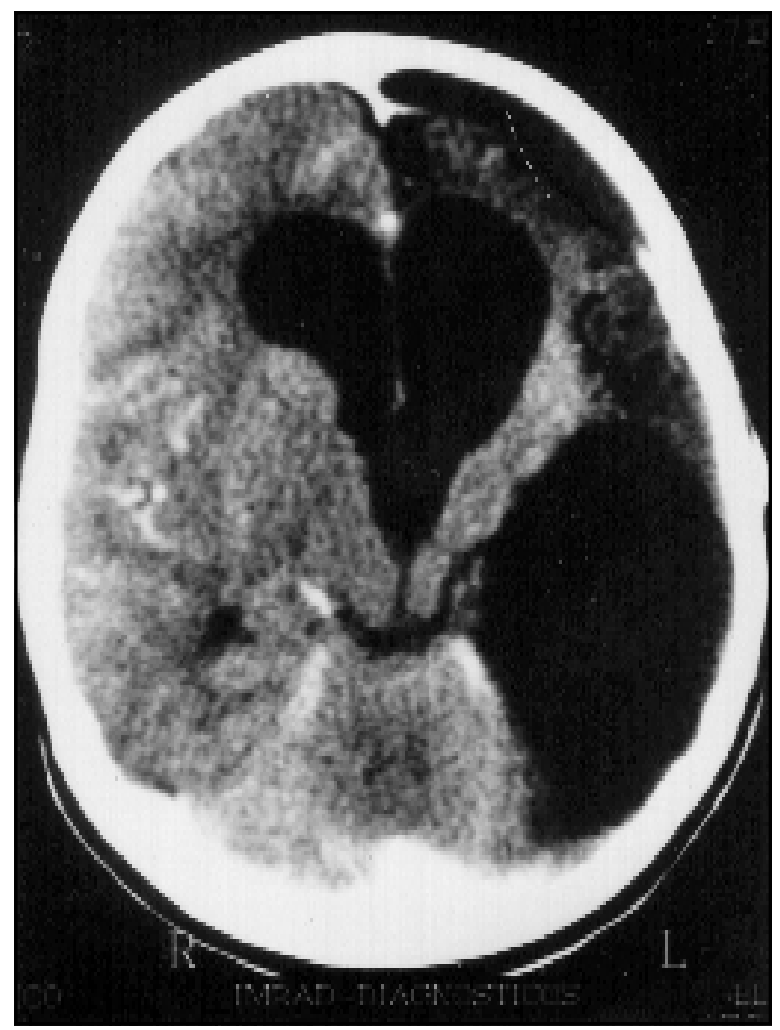

Fig 4. TC pós-operatória de paciente com carcinoma do plexo coróideo no ventrículo lateral, submetida a ressecção radical e com sobrevida superior a três anos.

liquórica permanente. Em nossa série de 15 pacientes, 11 (73,3\%) necessitaram de derivação liquórica, sendo dez no pré-operatório e uma no pós-operatório. Das quatro crianças que não necessitaram de derivações, duas tinham tumores no quarto ventrículo e duas nos ventrículos laterais, estão com acompanhamento respectivamente de 174, 31, 74 e 35 meses, todas foram submetidas a ressecção radical do tumor, sem tratamento complementar, três eram papilomas e um carcinoma. Alguns dados são interessante para confirmar que o tumor do plexo coróideo produz aumento na produção do líquor: encontramos, em nossa série, duas crianças que receberam uma derivação ventrículo-peritoneal com diagnóstico de hidrocefalia congênita e se apresentaram, meses depois, com volumosa ascite que desapareceu após o diagnóstico e exerese radical do tumor. A mesma observação já foi feita na literatura ${ }^{8,30}$; outra observação foi a saída de gotículas de líquor da superfície tumoral, em grande velocidade, o que nos levou a dizer que o tumor parecia uma "árvore de gotas d'água". Nas casuísticas contemporâneas ${ }^{3,8,28}$, o uso de derivações no pré-operatório tem sido frequente, o que demonstra não ser somente a hiperprodução liquórica a responsável pela hidrocefalia, como queria Matson ${ }^{4}$.

O tratamento dos tumores do plexo coróideo é essencialmente cirúrgico e sempre que possível a retirada do tumor deve ser em bloco e radical (Figs 3 e 4). Para que este objetivo seja alcançado, é importante a identificação do pedículo vascular e a ligadura da artéria nutridora e da veia de drenagem. A retirada radical e em bloco é sempre possível em tumores pequenos e médios e quando não existe invasão do parênquima cerebral, mesmo quando o diagnóstico e a cirurgia são feitas no récem- 
nascido $^{31,32}$. Nos tumores gigantes, principalmente em crianças com menos de seis meses ou quando o tumor invade o parênquima cerebral, na maioria das vezes se faz necessário o esvaziamento parcial do tumor antes de sua retirada total. Nestes casos deve-se ter controle rigoroso da perda sanguínea. Vraa-Jensen ${ }^{33}$ relata um caso isolado de metástase pulmonar em criança portadora de carcinoma do plexo coróideo. Sheridan e Besser ${ }^{34}$ relatam um caso de embolia pulmonar maciça, com óbito transoperatório, quando se tentava reduzir o volume do tumor; na descrição cirúrgica não se comenta sobre a ligadura prévia do pedículo venoso. As vias de acesso mais convencionais são a transcortical parieto-occipital para os tumores que ocupam os ventrículos laterais, a transcalosa para os tumores do terceiro ventrículo e a suboccipital para os tumores do quarto ventrículo. Atenção especial deve ser observada nos tumores que estão no quarto ventrículo e eventualmente invadem o tronco cerebral; o único óbito cirúrgico (até 30 dias após a cirurgia) da presente série ocorreu após a tentativa de se retirar o tumor que invadia o bulbo; a paciente apresentou dificuldade respiratória e paralisia de nervos bulbares, evoluindo com pneumonia de aspiração e óbito uma semana após a cirurgia.

A maioria dos tumores do plexo coróideo é constituída pelos papilomas, tumores benignos, geralmente confinados no interior dos ventrículos e passíveis de exerese radical e cura. O carcinoma do plexo coróideo corresponde de $20 \%$ a $40 \%{ }^{1,3,10,35,36}$ e representa de 1 a $3 \%$ dos tumores malignos em crianças ${ }^{5,36}$; segundo Duffner e col. ${ }^{7}$, em 70 a $85 \%$ dos casos acomete crianças com menos de 24 meses de idade. Na quase totalidade dos casos, o carcinoma do plexo coróideo em crianças é encontrado nos ventrículos laterais ${ }^{37,38}$. Excepcionalmente pode-se encontrar a hipertrofia do plexo coróideo e o cisto do plexo coróideo.

Enquanto o papiloma do plexo coróideo tem como opção a sua retirada radical levando à cura do paciente, o carcinoma tem ainda tratamento controverso. A radioterapia pré-operatória proposta por Carrea e Hawkins (citados por Tomita e $\mathrm{col}^{32}$ ), com o objetivo de reduzir o tamanho e o sangramento tumoral, está totalmente contraindicada em crianças. St.Clair ${ }^{10}$ propõe o tratamento quimioterápico após o diagnóstico anatomo-patológico colhido por biópsia, seguido de tratamento radical meses após. Dos quatro pacientes que foram submetidos ao protocolo de St.Clair ${ }^{10}$, um evoluiu para o óbito em 24 meses e os demais estavam vivos com 14, 30 e 39 meses, respectivamente; justifica o tratamento quimioterápico prévio pela redução importante no sangramento tornando possível uma ressecção mais ampla. Duffner e col. ${ }^{7}$, em estudo cooperativo aconselham ressecção ampla seguida de quimioterapia, sendo a radioterapia adiada, aguardando o crescimento da criança. $\mathrm{Na}$ experiência pessoal, foram duas as crianças com carcinoma do plexo coróideo (13,3\%) e nos dois casos foi conseguida a ressecção radical, com comprovação radiológica. Em uma das crianças foi observado, meses após, a disseminação tumoral pelas cisternas da fossa posterior, foi instituído tratamento quimioterápico mas a criança evoluiu para o óbito em 12 meses com metástases disseminadas pelo SNC; a segunda criança não tinha sinais de disseminação ou metástase, a ressecção foi total, não foi indicado tratamento complementar e controles tomográficos de seis em seis meses mostram ausência de recidiva local ou metástases, a criança esta com 35 meses de evolução.

\section{CONCLUSÃO}

O resultado final observado na literatura varia muito de acordo com a época em que foi apresentada a série. O prognóstico melhorou muito com a possibilidade de diagnóstico precoce por TC e RNM, progresso na neuroanestesia, técnicas microcirúrgicas e tratamento intensivo pediátrico no pós-operatório. Não existem dúvidas de que o tratamento do papiloma do plexo coróideo é a ressecção radical que pode ser conseguida em todos os casos, não havendo necessidade de tratamento complementar; já a conduta ideal frente ao carcinoma do plexo coróideo permanece nebulosa, mas tem-se que ter em mente que o tratamento é multidisciplinar com a cirurgia e tratamento oncológico associado. São necessários estudos cooperativos mais amplos, com protocolos rígidos, para que um número representativo de casos seja analisado e conclusões tiradas. 


\section{REFERÊNCIAS}

1. Aricó M, Raiteri E, Bossi G, et al. Choroid plexus carcinoma: report of one case with favourable response to treatment. Med Pediatr Oncol 1994;22:274-278.

2. Davis LE, Cushing H. Papillomas of the choroid plexus. Arch Neurol Psychiatr 1925;13:681-710.

3. Lena G, Genitori L, Molina J, Legatte JRS, Choux M. Choroid plexus tumours in children: review of 24 cases. Acta Neurochir 1990;106:68-72.

4. Matson DD, Crofton FDL. Papilloma of the choroid plexus in childhood. J Neurosurg 1960;17:1002-1027.

5. Paker RJ, Perilongo G, Johnson D, et al. Choroid plexus carcinoma of childhood. Cancer 1992;69:580-585.

6. Raimondi AJ, Gutierrez FA. Diagnosis and surgical treatment of choroid plexus papillomas. Child's Brain 1975;1:81-115.

7. Duffner PK, Kun LE, Burger PC, et al. Postoperative chemotherapy and delayed radiation in infants and very young children with choroid plexus carcinomas. Pediatr Neurosurg 1995;22:189-196.

8. Knierim DS. Choroid plexus tumors in infants. Pediatr Neurosurg 1990;16:276-280.

9. Tomita T, McLone DG. Brain tumors during the first twenty-four months of life. Neurosurgery 1985;17:913-919.

10. St.Clair SK, Humphreys RP, Pillay PK, Hoffman HJ, Blaser SI, Becker LE. Current management of choroid plexus carcinoma in children. Pediatr Neurosurg 1991;17:225-233.

11. Wilkins H, Rutledge BJ. Papillomas of the choroid plexus. J Neurosurg 1961;18:14-18.

12. Jooma R, Grant N. Third ventricle choroid plexus papillomas. Child's Brain 1983;10:242-250.

13. Matson DD. Hydrocephalus in a premature infant caused by papilloma of the choroid plexus. J Neurosurg 1953;10:416-420.

14. McGirr SJ, Ebersold MJ, Scheithauer BW, Quast LM, Shaw EG. Choroid plexus papillomas: long-term follow-up results in a surgically treated series. J Neurosurg 1988;69:843-849.

15. Nassar SI, Mount LA. Papillomas of the choroid plexus. J Neurosurg 1968;29:73-77.

16. Aicardi J, Lefébre J, Lerique-Koechlin A. A new syndrome: spasm in flexion, callosal agenesis, ocular abnormalities. Eletroencephalogr Clin Neurophysiol 1965;19:609-610.

17. Aguiar MFM, Cavalcanti M, Barbosa H, Vilela SL, Mendonça JL, Horta E. Sindrome de Aicardi associada a papiloma do plexo coróide: uma rara associação. Arq Neuropsiquiatr 1996;54:313-317.

18. Hamano K, Matsubara T, Shibata S, et al. Aicardi syndrome accompanied by auditory disturbance and multiple brain tumors. Brain Dev 1991;13:438-441.

19. Robinow M, Johnson GF, Minella PA. Aicardi syndrome, papilloma of the choroid plexus, cleft lip, and cleft of the posterior palate. J Pediatr 1984;104:404-405.

20. Chow PP, Horgan JG, Burns PN, Weltin G, Taylor KJW. Choroid plexus papilloma: detection by real-time and doppler sonography. AJNR 1986;7:168-170.

21. Pimenta AM, Pimenta LHM. Papiloma do plexo coróide em lactente de 9 meses sem hidrocefalia. Seara Med Neurocir 1981;10:291-296.

22. Sahar A, Feinsod M, Beller AJ. Choroid plexus papilloma: hydrocephalus and cerebrospinal fluid dynamics. Surg Neurol 1980;13:476-478.

23. Fairburn B. Choroid plexus papilloma and the relation to hydrocephalus. J Neurosurg 1960;17:166-171.

24. Ghatak NR, McWhorter JM. Ultrastrutural evidence for CSF production by a choroid plexus papilloma. J Neurosurg 1976;45:409-415.

25. Husag L, Costabile G, Probst C. Persistent hydrocephalus following removal of choroid plexus papilloma of the lateral ventricle. Neurochirurgia 1984;27:82-85.

26. Eisenberg HM, McComb JG, Lorenzo AV. Cerebrospinal fluid overproduction and hydrocephalus associated with choroid plexus papilloma. J Neurosurg 1974;40:381-385.

27. Gudeman SK, Sullivan HG, Rosner MJ, Becker DP. Surgical removal of bilateral papillomas of the choroid plexus of the lateral ventricles with resolution of hyfrocephalus. J Neurosurg 1979;50:677-681.

28. McDonald JV. Persistent hydrocephalus following the removal of papillomas of the choroid plexus of the lateral ventricle. J Neurosurg 1969;30:736-740.

29. Laurence KM, Hoare RD, Till K. The diagnosis of the choroid plexus papilloma of the lateral ventricle. Brain 1961;84:628-641.

30. Milhorat TH, Hammock MK, Chien T, Davis DA. Normal rate of cerebrospinal fluid formation five years after bilateral choroid plexectomy. J Neurosurg 1976;44:735-739.

31. Pascual-Castroviejo I, Roche MC, Villarejo F, Molina MG, Higueras AP. Choroid plexus papillomas of the fourth ventricle. Child"s Brain 1982;9:373-380.

32. Tomita T, Naidich TP. Successful resection of choroid plexus papillomas diagnosed at birth: report of two cases. Neurosurgery 1987;20:774-779.

33. Vraa-Jensen G. Papilloma of the choroid plexus with pulmonary metastases. Acta Psychiat Kbh 1950;25:299-306.

34. Sheridan M, Besser M. Fatal pulmonary embolism by tumor during resection of a choroid plexus papilloma: case report. Neurosurgery 1994;34:910-912.

35. Boyd MC, Steinbok P. Choroid plexus tumors: problems in diagnosis and management. J Neurosurg 1987;66:800-805.

36. Johnson DL. Management of choroid plexus tumors in children. Pediatr Neurosci 1989;15:195-206.

37. Carpenter DB, Michelsen J, Hays AP. Carcinoma of the choroid plexus. J Neurosurg 1982;56:722-727.

38. Dohrmann GJ, Collias JC. Choroid plexus carcinoma. J Neurosurg 1975;43:225-232. 